\title{
Rapid Raman Mapping for Chocolate Analysis
}

\author{
I. A. Larmour, K. Faulds and D. Graham* \\ Received (in $X X X, X X X)$ Xth $X X X X X X X X X 200 X$, Accepted Xth $X X X X X X X X X 200 X$ \\ First published on the web $X$ th $X X X X X X X X X 200 X$ \\ ${ }_{5}$ DOI: 10.1039/b000000x
}

Raman microspectroscopy mapping capabilities have advanced significantly and have been applied to cell and pharmaceutical tablet formulation analysis. Bulk Raman investigations of food and their constituents have been carried out but little work exists on the application of Raman mapping capabilities to food. Here, we assess the applicability of Raman microspectroscopy mapping to the

10 analysis of chocolate and examine both white and milk chocolate samples. It was found that the sucrose, lactose and fat constituents of white chocolate could be extracted and spatially resolved, indicating that the sucrose and lactose formed particles within a matrix of 'fats'. Fluorescence from cocoa solids present in milk chocolate prevented chemical mapping with the instrumentation used. Raman mapping should provide a powerful analytical technique for the analysis and

15 development of food products.

\section{Introduction}

Raman mapping can give a wealth of information about the spatial distribution and sizes of constituents within a complex sample. It has been used for the spatial analysis of active 20 pharmaceutical in the excipient matrix within a final tablet ${ }^{1-4}$ and has also been applied to in vivo studies where either a cell or a tissue section is analysed. ${ }^{5,6}$ The quality of data obtained has improved significantly as technological advances have been incorporated into the latest Raman microscope systems. ${ }_{25}$ Raman mapping can now be routinely carried out at a resolution set by the optical diffraction limit. However, one area where the possibilities of the information rich technique of Raman mapping has not yet been fully exploited, is within the food industry. ${ }^{7,8}$ Raman investigations of constituents and 30 final food products have been carried out by obtaining spectra at individual points. ${ }^{9-13}$ However, this approach provides low resolution data and gives no indication of particulate sizes or distribution within final products.

Since Cadbury's introduced the first chocolate bar in 1842 , 35 the global chocolate market has grown to an estimated value of $\$ 145 \mathrm{bn} /$ annum. ${ }^{14}$ Several analytical techniques have been applied to the analysis of chocolate or its constituents. Solid phase micro-extraction (SPME) followed by gas chromatograph toroidal ion trap mass spectrometry has been

40 used to analyse the volatile and non-volatile compounds in cocoa beans, cocoa butter and final chocolate products. ${ }^{15}$ The free fatty acids in chocolate were probed with liquid chromatograph - mass spectrometry (LC-MS) ${ }^{16}$ while the majority of reported work has been conducted with infrared 45 spectroscopy. ${ }^{17-20}$

Diffuse reflectance near-infrared Fourier transform spectroscopy (DRIFTS) was used to monitor the nutritional parameters of chocolate. ${ }^{17}$ Sucrose, lactose, fat and moisture levels within bulk chocolate or cocoa powder have all been 50 reported. ${ }^{18-20}$ However, to the best of our knowledge, there have been no reports in the primary literature of chemical mapping of chocolate.
Raman spectroscopy has been used to investigate the individual ingredients present within chocolate,,$^{9,21,22}$ as well 55 as their phase changes; ${ }^{10}$ however, the final product has not been the focus of analysis. We report the application of rapid Raman microspectroscopy mapping to final chocolate products. This technique provides a powerful development tool, not just for chocolate, but for the food industry as a 60 whole.

\section{Experimental}

\section{Materials}

The individual raw constituents were obtained from Mars Chocolate Ltd. These included; sucrose, lactose, whey, ${ }_{65}$ skimmed milk powder, anhydrous milk fat, vegetable fat, lecithin, cocoa butter and cocoa mass. Whey consists of $\sim 80 \%$ lactose, skimmed milk powder contains whey, lactose and anhydrous milk fat. Cocoa mass is approximately 50\% cocoa solids and 50\% cocoa butter. Final preparation white and milk 70 chocolate samples were also provided. All materials were used without further purification.

\section{Instrumentation}

A WITec Alpha 300R confocal Raman microscope was used for all spectral acquisitions with independent spectrographs 75 for the two laser wavelengths investigated; 532 and $785 \mathrm{~nm}$. The gratings were $600 \mathrm{~g} / \mathrm{mm}(\mathrm{BLZ}=500 \mathrm{~nm})$ and $300 \mathrm{~g} / \mathrm{mm}$ $(B L Z=750 \mathrm{~nm})$ and were coupled to thermoelectrically cooled charge-coupled devices (CCDs) which gave spectral resolutions of $4 \mathrm{~cm}^{-1}$ and $3.7 \mathrm{~cm}^{-1}$ respectively across a 80 spectral range of $3936 \mathrm{~cm}^{-1}$ and $3324 \mathrm{~cm}^{-1}$. Unfocussed laser powers at the sample were measured at $\sim 37 \mathrm{~mW}$ and $\sim 80 \mathrm{~mW}$ respectively.

Reference and map spectra were recorded using an Olympus LMPlanFI 50x/0.50 long working distance objective. ${ }_{85}$ The cocoa mass reference had to be recorded using a Nikon EPlan 10x/0.25 LWD objective and accumulated for 5 seconds to prevent sample degradation and detector 
saturation. All reference spectra of the remaining pure constituents were accumulated for 60 seconds.

High resolution mapping was carried out on an area measuring $80 \times 80 \mu \mathrm{m} .160$ points/line and 160 lines/image 5 were recorded to give a resolution of $0.5 \mu \mathrm{m}$. Spectra were recorded for $50 \mathrm{~ms}$ per point. Laser powers were reduced for milk chocolate samples by means of attenuators on the fibre optic coupling on the laser heads. Data analysis was carried out using WITec Project software, version 2.02.

\section{${ }_{10}$ Results and Discussion}

Using $532 \mathrm{~nm}$ laser excitation it was found that skimmed milk powder, cocoa mass, whey and lecithin (an emulsifier) all fluoresced, figure S1 and contained no vibrational spectral features suitable for component identification. The other raw 15 ingredients produced spectra with good signal-to-noise levels. These constituents contained vibrational bands that could be used for component extraction, figure 1.

The band at $636 \mathrm{~cm}^{-1}$ was used for identification of sucrose, while the band at $468 \mathrm{~cm}^{-1}$ identified lactose. Vegetable fat, 20 anhydrous milk fat and cocoa butter all showed very similar spectral features and were therefore classified generally as 'fats' with the band at $1295 \mathrm{~cm}^{-1}$, assigned to $-\mathrm{CH}_{2}$ deformation, ${ }^{9}$ used for identification.

Initial mapping experiments were performed on white 25 chocolate samples due to the reduced risk of sample degradation. It was found that spectra could be recorded at the maximum laser power without observable sample damage occurring. Figure 2 shows the individual Raman maps extracted for each of the three main components; sucrose, 30 lactose and 'fats', using the bands identified in figure 1 . The combined map is also shown, which clearly shows that the sucrose and lactose particles were found within a matrix of 'fats'. These maps contain 25,600 individual full range spectra and were recorded in approximately 40 minutes. The time 35 discrepancy is largely due to stage movements.

Raman mapping did not only give spatial distribution information about the constituents, it also provided sizing information. In the example shown the lactose and sucrose particles were measured to be $4.9 \pm 2.1 \mu \mathrm{m}$ and $4.4 \pm 1.5 \mu \mathrm{m}$ 40 respectively. This was in agreement with the particle sizes of the raw materials measured via light scattering and the final crystal sizes observed under electron microscopy (data not shown), although significantly it gave non-destructive, unambiguous chemical information of what the crystals were 45 made from within the final product.

Milk chocolate samples proved significantly more difficult to map due to sample damage from the laser excitation. The unfocussed laser power at the sample had to be reduced to $<$ $0.1 \mathrm{~mW}$ to prevent degradation of the chocolate surface. Once so sample degradation was minimised sample fluorescence became the overriding problem. It has already been mentioned that skimmed milk powder, cocoa mass, whey and lecithin all fluoresce at $532 \mathrm{~nm}$. However, cocoa mass was responsible for the observed fluorescence as this was the only fluorescent 55 constituent missing from white chocolate, in which no problems with sample fluorescence were observed. Cocoa mass contains cocoa butter and cocoa solids. Since, the spectra from cocoa butter has already been recorded and showed no fluorescence, this means that the detrimental 60 fluorescence was obtained from the cocoa solids component. It should be noted that although skimmed milk powder, whey and lecithin are used within white chocolate their fluorescent signals did not overwhelm the signals from the other constituents.

65 In an attempt to extend Raman mapping to milk chocolate samples the wavelength was increased to $785 \mathrm{~nm}$. Figure 3, shows the spectra recorded from the non-fluorescent constituents. Lactose, sucrose and the 'fats' could be discriminated with the same bands as those used for the 532 $70 \mathrm{~nm}$ results, this resulted in similar maps being produced for white chocolate, indicating no wavelength dependence of the results, figure S2. Skimmed milk powder and whey did not give fluorescent signals, although they did not contain individual vibrational bands which could be used for their 75 discrimination without further data analysis such as modified alternating least squares regression. ${ }^{23}$ Unfortunately, cocoa mass and lecithin continued to be fluorescent at this wavelength.

Milk chocolate mapping at $785 \mathrm{~nm}$ suffered from laser 80 induced sample damage and the power had to be significantly reduced from $80 \mathrm{~mW}$ to $\sim 2 \mathrm{~mW}$ to ensure no observable sample degradation occurred. Figure 4 shows the map extracted using the lactose peak, it shows some structural features however, these turned out to be fluctuations within 85 the fluorescence signal rather than any 'real' features. Cocoa solids are again responsible for the poor Raman mapping of milk chocolate. We have observed that cocoa mass, containing cocoa solids, continues to show a fluorescent response, even when $830 \mathrm{~nm}$ laser excitation is used (data not shown). 90 Therefore, either Raman mapping using longer wavelengths, such as $1064 \mathrm{~nm}$, is required or more sophisticated fluorescence rejection techniques employed. Although it has not been possible to map milk chocolate samples, we have successfully demonstrated Raman mapping of white chocolate 95 samples.

\section{Conclusions}

We have successfully applied rapid, high-resolution, largearea, Raman mapping to the analysis of chocolate samples. White chocolate was mapped with no special sample or 100 instrumental alterations and showed that lactose and sucrose particles were embedded in a matrix of 'fats'. Laser powers had to be significantly reduced to prevent sample damage with respect to milk chocolate samples. Milk chocolate mapping also suffered due to inherent fluorescence from the cocoa 105 solid constituent. Importantly, Raman mapping did not only provide spatial distribution of constituents, it also allowed the particles to be sized and their chemical nature to be assigned unambiguously. Raman microspectroscopy mapping should become a significant addition to the food analysts' toolkit.

\section{${ }_{110}$ Notes and references}

Centre for Molecular Nanometrology, WestCHEM, Department of Pure and Applied Chemistry, University of Strathclyde, 295 Cathedral Street, 
Glasgow, UK. E-mail: duncan.graham@strath.ac.uk; Tel: +44 141548 4701

$\dagger$ Electronic Supplementary Information (ESI) available: Fluorescent constituents at $532 \mathrm{~nm}$ and $785 \mathrm{~nm}$ maps. See DOI: 10.1039/b000000x/

51 C. Gendrin; Y. Roggo and C. Collet, J. Pharm. Biomed. Anal., 2008, 48, 533.

2 M. J. Henson and L. Zhang, Appl. Spectrosc., 2006, 60, 1247.

3 S. Sasic, Appl. Spectrosc., 2007, 61, 239.

4 S. Wartewig and R. H. H. Neubert, Adv. Drug Delivery Rev., 2005, 57, 1144 .

5 J. R. Beattie; S. Brockbank; J. J. McGarvey and W. J. Curry, Mol. Vis., 2007, 13, 1106.

6 C. Krafft; T. Knetschke; A. Siegner; R. H. W. Funk and R. Salzer, Vib. Spectrosc., 2003, 32, 75.

157 P. D. A. Pudney; T. M. Hancewicz and D. G. Cunningham, Spectr.Int. J., 2002, 16, 217.

8 P. D. A. Pudney; T. M. Hancewicz; D. G. Cunningham and M. C. Brown, Vib. Spectrosc., 2004, 34, 123.

9 V. Baeten; P. Hourant; M. T. Morales and R. Aparicio, J. Agric.

$20 \quad$ Food Chem., 1998, 46, 2638.

10 A. Celedon and J. M. Aguilera, Food Sci. Tech. Int., 2002, 8, 101.

11 L. F. C. de Oliveira; R. Colombara and H. G. M. Edwards, Appl. Spectrosc., 2002, 56, 306.

12 J. R. Beattie; S. E. J. Bell; C. Borgaard; A. M. Fearon and B. W. Moss, Lipids, 2004, 39, 897

13 S. Okazaki; M. Hiramatsu; K. Gonmori; O. Suzuki and A. T. Tu, Forensic Toxicol., 2009, 27, 94.

14 Leatherhead Food Research, "The Global Confectionery Market Trends and Innovations," 2006, pp. 212.

3015 C. R. Bowerbank; A. McShea; S. B. Munro; E. D. Lee and D. W. Later, Lc Gc N. Am., 2009, 31.

16 D. Perret; A. Gentili; S. Marchese; M. Sergi and L. Caporossi, Rapid Commun. Mass Spectrom., 2004, 18, 1989.

17 J. Moros; F. A. Inon; S. Garrigues and M. de la Guardia, Anal. Chim. Acta, 2007, 584, 215

18 P. A. da Costa Filho, Anal. Chim. Acta, 2009, 631, 206.

19 A. Vesela; A. S. Barros; A. Synytsya; I. Delgadillo; J. Copikova and M. A. Coimbra, Anal. Chim. Acta, 2007, 601, 77.

20 J. Tarkosova and J. Copikova, J. Near Infrared Spectrosc., 2000, 8, $40 \quad 251$.

21 M. Liang; V. T. Chen; H. L. Chen and W. L. Chen, Talanta, 2006, 69, 1269.

22 H. G. M. Edwards; S. E. J. Villar; L. F. C. de Oliveira and M. L. Hyaric, Anal. Chim. Acta, 2005, 538, 175.

4523 J. H. Wang; P. K. Hopke; T. M. Hancewicz and S. L. L. Zhang, Anal. Chim. Acta, 2003, 476, 93

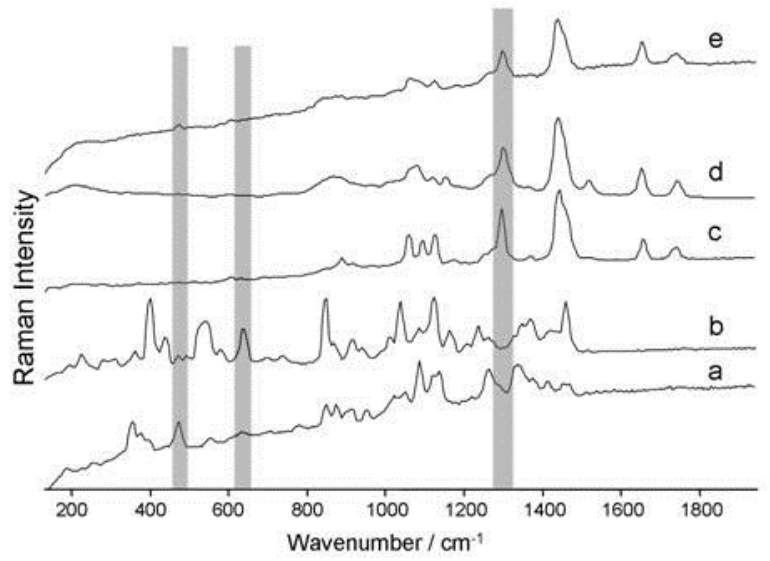

Fig. 1 Reference spectra of the non-fluorescent constituents of chocolate at $532 \mathrm{~nm}$, accumulation time of 60 seconds. a - lactose, $\mathrm{b}$ - sucrose, $\mathrm{c}-$ 50 cocoa butter, $\mathrm{d}$ - anhydrous milk fat, $\mathrm{e}-$ vegetable fat. The bands used for identification of the lactose, sucrose and 'fats' are identified.

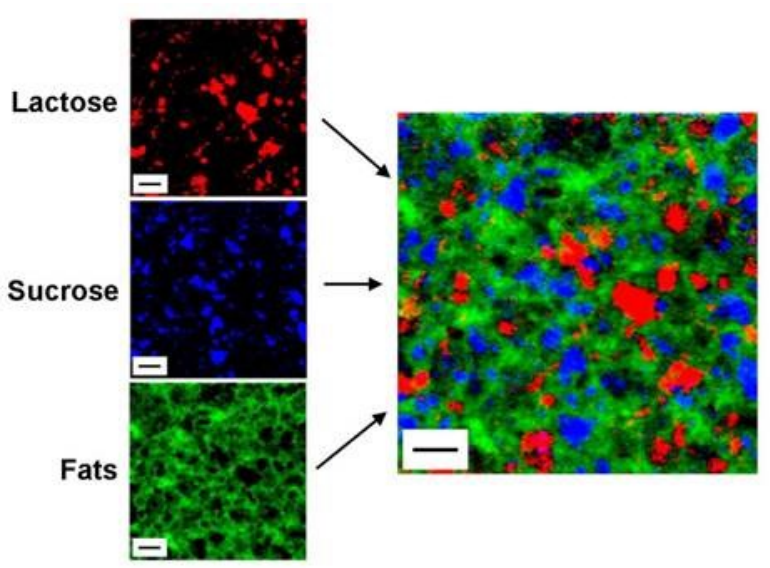

Fig. 2 Raman mapping of a white chocolate sample measured at $532 \mathrm{~nm}$, constituent maps and the combined Raman map which shows the lactose 55 and sucrose particles within a matrix of 'fats'. Scale bar in each image is $10 \mu \mathrm{m}$.

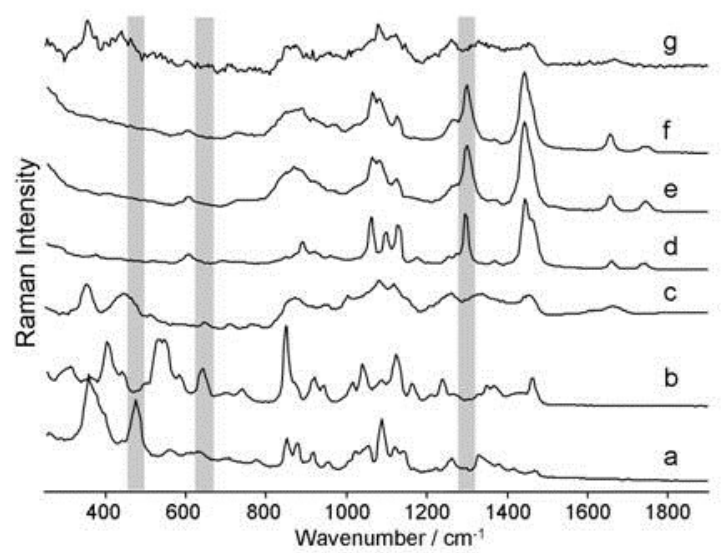

Fig. 3 Reference spectra of the non-fluorescent chocolate constituents measured at $785 \mathrm{~nm}$. a - lactose, $\mathrm{b}$ - sucrose, $\mathrm{c}$ - skimmed milk powder, d $60-$ cocoa butter, $\mathrm{e}-$ anhydrous milk fat, $\mathrm{f}-$ vegetable fat, $\mathrm{g}-$ whey. All spectra were accumulated for 60 seconds. The bands used for identification of the lactose, sucrose and 'fats' are identified.

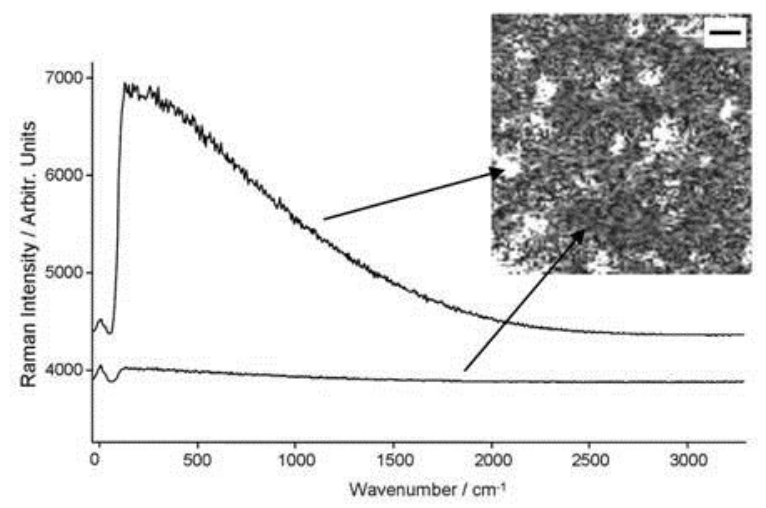

Fig. 4 An extracted 'lactose' map from a milk chocolate sample showing 65 features. These turned out to be variations within the fluorescence signal intensity as shown. Scale bar in the map is $10 \mu \mathrm{m}$. 


\section{$\underline{\text { Supplementary Information }}$}

\section{Rapid Raman Mapping for Chocolate Analysis}

I. A. Larmour, K. Faulds and D. Graham*

Centre for Molecular Nanometrology, WestCHEM, Department of Pure and Applied Chemistry, University of Strathclyde, 295 Cathedral Street, Glasgow, UK. E-mail: duncan.graham@strath.ac.uk; Tel: +441415484701

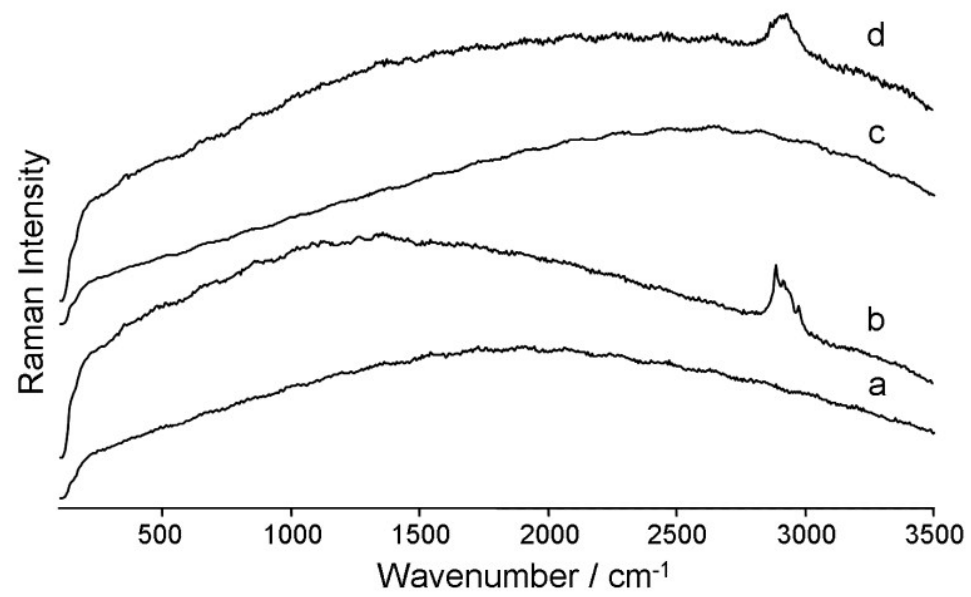

Figure S1. Reference spectra of the constituents which showed fluorescence at 532 $\mathrm{nm}$ laser excitation. $\mathrm{a}$ - lecithin, $\mathrm{b}$ - whey, $\mathrm{c}$ - cocoa mass, $\mathrm{d}$ - skimmed milk powder. Spectra were accumulated for 60 seconds apart from $\mathrm{c}$ which was accumulated for 5 seconds with a 10x objective, see main text.

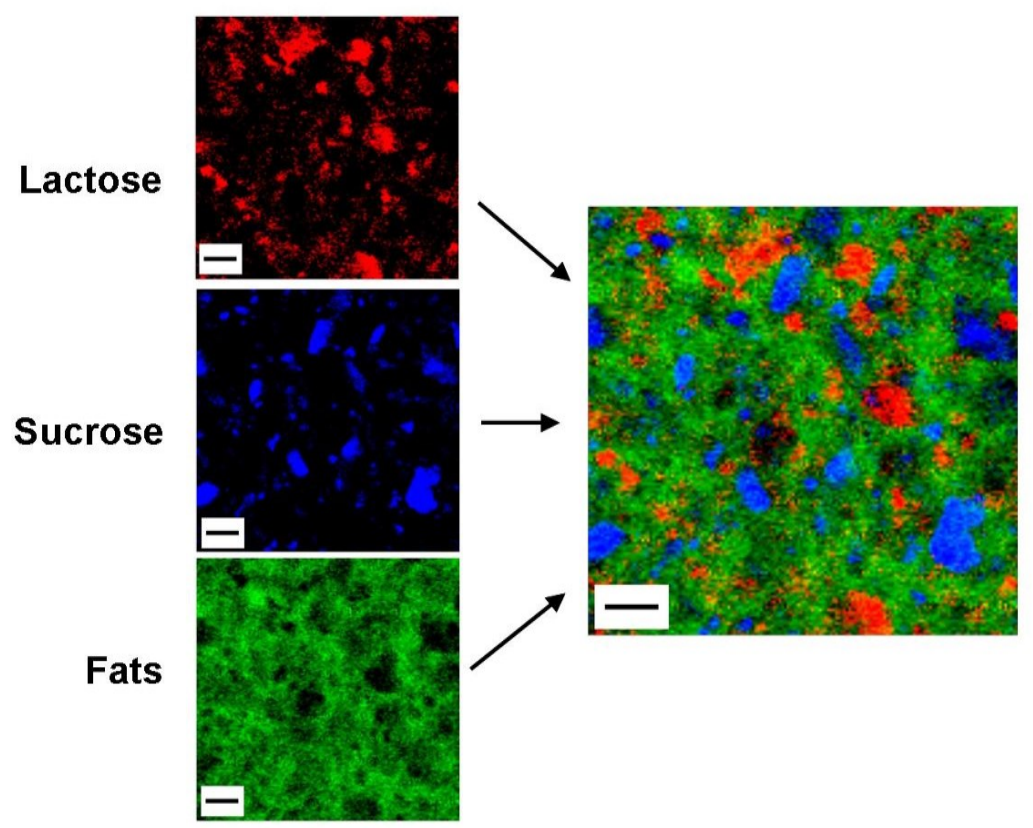

Figure S2. Raman mapping of a white chocolate sample measured at $785 \mathrm{~nm}$, constituent maps and the combined Raman map which shows the lactose and sucrose particles within a matrix of 'fats'. Scale bar in each image is $10 \mu \mathrm{m}$. 